\title{
Present and Future of Pediatric Hemophilia Treatment
}

\section{Giuseppe Lassandro $^{1}$, Anna Amoruso ${ }^{1}$ and Paola Giordano ${ }^{*}$}

Department of Biomedical Science and Human Oncology, Pediatric Unit University of Bari "A. Moro", Bari, Italy

Hemophilia is the most common X-linked inherited bleeding disorder caused by a deficiency of coagulation factor VIII (in hemophilia A) or factor IX (in hemophlia B). This deficiency is the result of mutations of the respective clotting factor genes. Hemophilia $\mathrm{A}$ is more common than hemophilia B representing $80-85 \%$ of the total hemophilia population. The peculiarity in hemophilia is the bleeding tendency: the severity of bleeding is generally correlated with the clotting factor level [1].

There are three forms of hemophilia:

- $\quad$ Severe, with factor $<1 \%$

- Moderate, with factor 1-5\%

- $\quad$ Mild, with factor $>5 \%$ and $<40 \%$

The principal clinical manifestations are hematomas and haemarthroses: the sites of bleeding in hemophilia are muscles (in particular hematoma of the iliopsoas muscles) and some joints (ankles, knees, elbows, and wrist). The diagnosis of hemophilia can be suspected in patients with spontaneous bleeding or with a family history of bleeding or bruising in early childhood or with the finding of an aPTT extended and then the diagnosis is confirmed by the deficiency of the clotting factor [2]. Quality of life has become in the western countries the primary objective of the process of providing care in hemophilia population and for this reason the principal aim of care is to prevent and treat bleeding with the deficient clotting factor [3]. Currently, the most challenging complication of therapy has become the development of inhibitory alloantibodies against FVIII or FIX. These inhibitors, that develop in $25-30 \%$ of severe hemophilia A patients and in only $3-5 \%$ of those with hemophilia B, render replacement therapies ineffective. The development of inhibitory all antibodies is higher in the first few doses of exogenous coagulation factor and it is reduced after 150 doses [4]. The introduction of bypassing agents, such as activated prothrombin complex concentrates (APCC) (Factor Eight Inhibitor Bypassing Activity-FEIBA) and recombinant activated factor VII (rFVII, NovoSeven), has improved the management of children with inhibitors. Currently, inhibitors are the most severe complication and prophylasix is emerging as the optimal preventive care strategy [5]. The therapy of hemophilia can be on a treatment "on demand" or "on a regimen of prophylaxis": the therapy on demand is based on the intravenous administration of clotting factor during the hemorrhagic event and the dose of clotting factor depends on the severity of the bleeding while prophylaxis is the treatment by intravenous injection of factor concentrate to prevent anticipated bleeding and it is the gold standard for preserving joint function in babies with severe hemophila. We can talk about three forms of prophylaxis: primary prophylaxis is defined as regular treatment, given for at least 45 weeks of the year, initiated in the absence of documented osteochondral joint disease, determined by physical examination and/or imaging studies, and started before the second clinically evident large joint bleed and age 3 years; secondary prophylaxis is defined as regular treatment started after two or more bleeds into ankles, knees, hips, elbows or shoulders and before the onset of joint disease documented by physical examination and imaging studies, given for at least 45 weeks of the year; tertiary prophylaxis is described as regular continuous treatment started after the onset of joint disease documented by physical examination and plain radiographs of the affected joints [4]. The goal of prophylaxis is

\section{Publication History:}

Received: December 15, 2015

Accepted: February 06, 2016

Published: February 09, 2016

\section{Keywords:}

Neonatal Seizures, Hemophilia, Metabolism

is to maintain a clotting factor concentration $>1 \%$ of normal at all times so as to avoid breakthrough bleeds. To do so usually requires the administration of FVIII $15-30 \mathrm{UI} / \mathrm{kg} /$ daily or $20-40 \mathrm{UI} / \mathrm{kg}$ every second day or at least three times weekly for patients with haemophilia A and every third day or twice weekly for patients with haemophilia B. One option for the treatment of very young children is to start prophylaxis once a week and escalate depending on bleeding and venous access. Prophylaxis is best given in the morning to cover periods of activity. Prophylactic administration of clotting factor concentrates is advisable prior to engaging in activities with higher risk of injury [6]. The treatment can be based on plasma-derived clotting factor concentrates obtained from human plasma, which currently they are little used; or on recombinant drugs, obtained in the laboratory and nowadays the most used. They are classified according to the content of human or animal proteins in the culture medium or in the final formulation. The production of recombinant coagulation factors have increased the worldwide capacity for replacement therapy and facilitated aggressive prophylactic therapy [4]. Recombinant DNA technology remains a promising platform to develop novel hemophilia therapeutics with improved functional properties to try to overcome some barriers that still persist. Bioengineering strategies have produced novel therapeutics with increased production efficiency, increased potency and resistance to inactivation, prolonged plasma half-lives, and reduced immunogenicity. Purified plasmaderived factor VIII (pdFVIII) concentrates have been available since the 1970s, but require rigorous plasma donor screening and viral inactivation technologies to reduce the risk for blood-transmitted pathogens. Recombinant FVIII (rFVIII) concentrate has proven to be a remarkable facsimile to pdFVIII with regard to biochemical and hemostatic properties, and has a similar pharmacokinetic profile with a half-life of roughly 12 hours [7]. After almost 20 years of experience with rFVIII, there are clearly opportunities to extend recombinant DNA technology to further enhance replacement therapy. Bioengineering strategies have been directed at overcoming the inherent limitations of rFVIII biosynthesis and secretion, functional activity, half-life, and antigenicity/immunogenicity. Some of these strategies have already reached commercialization. It was demonstrated that the B domain of FVIII, the equivalent of approximately $38 \%$ of the primary cDNA sequence, could be removed without loss of FVIII procoagulant activity.

"Corresponding Author: Dr. Paola Giordano, Department of Biomedical Science and Human Oncology, Pediatric Unit, University of Bari "Aldo Moro", Bari, Italy; E-mail: paola.giordano@uniba.it

Citation: Lassandro G, Amoruso A, Giordano P (2016) Neonatal Seizures and Inborn Errors of Metabolism: An Update. Int J Pediatr Neonat Care 2: 112. doi: http://dx.doi.org/10.15344/2455-2364/2016/112

Copyright: (C) 2016 Lassandro et al. This is an open-access article distributed under the terms of the Creative Commons Attribution License, which permits unrestricted use, distribution, and reproduction in any medium, provided the original author and source are credited. 
This significantly improved the yield of rFVIII due to markedly increased levels of mRNA and increased translation. BDD-rFVIII remains the first and only modified human rFVIII molecule to come to commercialization. Other strategies are based on the extension of half-life in fact prolonging the half-life of FVIII could greatly reduce the frequency and dose of infusions, thereby improving the efficacy of prophylaxis through better compliance, as well as improve convenience and patient quality of life [8]. Some of the half-life extension strategies are sustained delivery through association of rFVIII with polyethylene glycol (PEG)ylated liposomes, chemical modification (PEGylation, polysialylation), bioengineering rFVIII through mutagenesis at putative binding sites for clearance receptors, or the generation of fusion proteins. The primary advantage of PEGylation for a biologic is the incorporation of many water molecules within the hydrophilic PEG structures, functionally increasing the effective size of the conjugated protein above the filtration size of the kidney. This is of no particular advantage for FVIII, because it is already too large for kidney filtration. Any advantage to PEGylation of FVIII is likely through disruption of interaction with cellular clearance receptors [9]. However, such chemical modification could be a disadvantage to FVIII if it interfered with key protein-protein interactions (vWF, thrombin, and activated FIX). These potential hazards of chemical modification of FVIII are particularly problematic without the ability to target the sites where PEG polymers are conjugated to the protein. Polysialic acid (PSA) modification of therapeutic proteins is an alternative strategy to PEGylation for increasing the size of a protein. Some of the advantages that have been demonstrated with PSA technology include reduced immunogenicity and antigenicity, and preservation of function with increased stability. Furthermore, PSA, unlike PEG, are naturally occurring and biodegradable, which offers a potential advantage when administering large doses of a biologic therapy over a prolonged period of time. PSA modification is now being explored through direct conjugation of rFVIII, FIX, FVIIa, and vWF [7]. Formulation with PEGylated liposomes is an established process that has been used to extend the half-life of a broad range of therapeutic proteins, including FVIII. For this approach, the therapeutic protein is reconstituted with PEGylated liposomes as a carrier. Because liposomes are typically cleared very quickly from the circulation, the addition of PEG can extend the circulatory half-life of the liposomes considerably. This approach can effectively modify pharmacokinetic and pharmacodynamic properties of proteins and has been utilized to develop a potentially longer acting FVIII. With this strategy, rFVIII molecules remain unmodified, so there is no loss of normal protein-protein interactions and functional activities. Another line of investigation involves developing FVIII with reduced antigenicity/immunogenicity. The most sophisticated constructions are human/porcine hybrid BDD-rFVIII molecules. As regards the coagulation factor IX, purifiedpdFIXhasbeenavailabl esince1992, followed by recombinant FIX (rFIX) in 1998, produced in mammalian cell lines similar to rFVIII. Although, rFIX also shares nearly identical hemostatic properties with pdFIX, there are differences in post translation modifications of rFIX that result in altered pharmacokinetics. Specifically, rFIX exhibits an 30\% reduced recovery in plasma at equivalent dosing to pdFIX, requiring dose modification for treatment and prophylactic regimens. However, the plasma half-life for both pdFIX and rFIX is similar at roughly 16 hours. Alternative Expression Systems An innovative expression technology has been used to enhance the expression of rFIX in stable cell lines. This strategy involves flanking rFIX cDNA with the transcriptional control regions from Chinese hamster elongation factor $1 \alpha$, a highly expressed gene within Chinese hamster ovary
(CHO) cells. Within this system, the expression levels of rFIX are increased 10-fold over traditional stably transfected $\mathrm{CHO}$ cells. Such technology could be exploitedfor efficient production of rFIX, or any other recombinant protein, and hopefully facilitate reduced costs. Several bioengineering strategies have generated $\mathrm{rFIX}$ variants with increased potency. These include point mutations, such as variant FIX-R338A, which exhibits 3- to 7-fold higher specific activity in vitro and in vivo. Strategies to extend the half-life of FIX include direct, targeted modification with PEGylation and fusion protein technology, which links FIX to another protein with a much longer plasma halflife. rFIX was fused to the constant region $(\mathrm{Fc})$ of immunoglobulin $\mathrm{G}$ $(\mathrm{rFIXFc})$. The presence of the Fc portion protects the fusion protein from catabolism through interaction with the neonatal $\mathrm{Fc}$ receptor (FcRn) [3]. In a second strategy, rFIX was fused to albumin (rFIXFP) via a cleavable peptide linker. New frontiers may be represented by non-intravenous infusions: for example oral, intratracheal, subcutaneous an intramuscular administration because they could free patients from the problem of regular venous access and from the complications of central venous catheters. Research toward novel therapeutics for hemophilia is not limited to bioengineering recombinant coagulation proteins. Alternative protein and nonprotein agents are showing promise in preclinical development [10]. Kopecky et al synthetically designed peptide sequences that were screened and tested for their ability to bind to inhibitory antibodies effectively neutralizing anti-FVIII antibodies in vitro. The promising peptides typically have sequences that are similar to FVIII. Fucoidans, known also as non anticoagulant-sulfated polysaccharides (NASP), are heparin-like molecules that at certain concentrations exhibit hemostatic properties rather than anticoagulant activity. They are believed to act through blockade of tissue factor pathway inhibitor, effectively removing the brakes from coagulation. NASP can accelerate the clotting times of plasma from hemophilia patients [11]. Finally, missense mutations leading to premature stop codons in FVIII or FIX occur in approximately $10 \%$ to $15 \%$ of persons with hemophilia A or B, respectively. Small molecules have been developed that can facilitate translational read through of premature stop codons $[7,12]$. In conclusion we can say that the research and innovation strategies are moving forward so that we can ensure an optimal quality of life. Quality of life has become in the western countries the primary objective of the process of providing care. The hemophiliac child of the new millenniumis, in western countries, a child that can have treatment options that provide to live a childhood almost similar to that of their peers, and therefore must socialize and live in community. He's a child that can and should safety engage in physical activity. The child with hemophilia must be adequately informed of his conditions that he can manage his path toward the adult age through school and work. The role of the pediatrician is therefore crucial because it must, clutching a bond of trust with the child, be able to coordinate all caregivers to accompany his growth step by step [6].

\section{References}

1. Bolton-Maggs PH, Pasi KJ (2003) Haemophilias A and B. Lancet 361 1801-1809.

2. Franchini M, Mannucci PM (2012) Past, present and future of hemophilia: a narrative review. Orphanet J Rare Dis 7: 24 .

3. Giordano P, Lassandro G, Valente M, Molinari AC, leranò P, et al. (2014) Current management of the hemophilic child: a demanding interlocutor. Quality of life and adequate cost-efficacy analysis. Pediatr Hematol Oncol. 31: 687-702.

4. Srivastava A, Brewer AK, Mauser-Bunschoten EP, Key NS, Kitchen S, et al. (2013) Guidelines for the management of hemophilia. Haemophilia 19: e1-47. 
Citation: Lassandro G, Amoruso A, Giordano P (2016) Neonatal Seizures and Inborn Errors of Metabolism: An Update. Int J Pediatr Neonat Care 2: 112. doi: http://dx.doi.org/10.15344/2455-2364/2016/112

Page 3 of 3

5. Franchini M, Mannucci PM (2011) Inhibitors of propagation of coagulation (factors VIII, IX and XI): a review of current therapeutic practice. Br J Clin Pharmacol 72: 553-562.

6. Giordano P, Franchini M, Lassandro G, Faienza MF, Valente R, et al. (2013) Issues in pediatric haemophilia care. Ital J Pediatr 39: 24.

7. Pipe SW (2010) Hemophilia: new protein therapeutics. Hematology Am Soc Hematol Educ Program 2010: 203-209.

8. Iantier JL, Rodriguez MH, Enjolras N, Attali O, Negrier C (2001) A factor VII minigene comprising the truncated intron I of factorlX highly improves the in vitro production of factor VIII. ThrombHaemost 86: 596-603.

9. Powell JS, Nugent DJ, Harrison JA, Soni A, Luk A, et al. (2008) Safety and pharmacokinetics of a recombinant factor VIII with pegylated liposomes in severe hemophilia A. J Thromb Haemost 6: 277-283.

10. Metzner HJ, Weimer T, Kronthaler U, Lang W, Schulte S (2009) Genetic fusion to albumin improves the pharmacokinetic properties of factor IX. Thromb Haemost 102: 634-644

11. Liu T, Scallan CD, Broze GJ Jr, Patarroyo-White S, Pierce GF, et al. (2006) Improved coagulation in bleeding disorders bynon-anticoagulant sulfated polysaccharides (NASP). Thromb Haemost 95: 68-76.

12. White RR, Sullenger BA, Rusconi CP (2000) Developing aptamers into therapeutics. J Clin Invest 106: 929-934. 